\title{
Finite-Time Synchronization of Fractional-Order Chaotic Systems with Different Structures under Stochastic Disturbances
}

\author{
Weiqiu Pan, Tianzeng $\mathrm{Li}^{*}$ \\ School of Mathematics and Statistics, Sichuan University of Science and Engineering, Zigong, China \\ Email: 2396269767@qq.com, *litianzeng27@163.com
}

How to cite this paper: Pan, W.Q. and $\mathrm{Li}$, T.Z. (2021) Finite-Time Synchronization of Fractional-Order Chaotic Systems with Different Structures under Stochastic Disturbances. Journal of Computer and Communications, 9, 120-137.

https://doi.org/10.4236/jcc.2021.96007

Received: May 9, 2021

Accepted: June 18, 2021

Published: June 21, 2021

Copyright $\odot 2021$ by author(s) and Scientific Research Publishing Inc. This work is licensed under the Creative Commons Attribution International License (CC BY 4.0).

http://creativecommons.org/licenses/by/4.0/

\section{(c) (i) Open Access}

\begin{abstract}
This paper studies the finite-time synchronization of fractional-order chaotic systems with different structures under parameter disturbance and external disturbance. We put forward a fractional-order controller that can achieve the finite-time synchronization of any-order fractional-order chaotic systems under stochastic disturbances. This controller has good robustness and anti-interference performance. With the concept of the finite-time stability theory given, some judgment criterions for the synchronization of fractional-order chaotic systems are proved. This method can not only make the error systems have a faster convergence rate but also can be implemented in engineering easily. The numerical simulations of two specific examples demonstrate the effectiveness of the method. At the same time, the synchronised time of finite-time synchronization is shorter and faster than the complete synchronization and the time can be adjusted according to the parameters in the controller.
\end{abstract}

\section{Keywords}

Fractional Order, Chaos, Finite-Time Synchronization, Stochastic

Disturbance

\section{Introduction}

Although the development history of fractional and integer calculus is not much different, it turns out that fractional calculus is of great significance to express the model we are studying. This is because it not only has the memory function 
but also more accurately describes the fractional order dynamic models. Of course, fractional calculus has many other advantages [1] [2] [3]. It's because of these benefits that the theories of fractional calculus are widely used in physics [4], engineering [2], chemistry [5] and other subjects. In recent decades, these fields including information processing [6], secure communication [7], thermal systems [8], robot control and problems [9] have been discovered that many physical processes exhibit fractional order dynamic behavior.

Chaos has achieved tremendous and far-reaching development in many fields after discovering the first chaotic attractor by Lorenz. At present, the research on chaos control and synchronization has spread across many disciplines. In the past 20 years, many methods of chaos control have appeared, such as OGY control [10], drive-response control [11], pulse control [12], sliding mode control [13], active control [14], Lyapunov direct method [15], etc. Taking into account above methods, some scholars have considered the synchronization problem for a class of fractional-order chaotic systems [16] [17] [18] while others only concentrate on the specific chaotic systems [19] [20] [21]. And many scholars have solved the synchronization problem of chaotic systems of any order [22] [23].

Currently, the main research direction is to make the system reach synchronization in infinite time, that is, to consider the asymptotically stable of systems. However, facing the actual situation, we may need a specified time, that is, finite-time synchronization. It not only has a faster convergence rate but also stronger robustness [24]. Keyong S et al. used the finite-time synchronization theories to define a nonlinear controller which realizes that the synchronization of four-dimensional fractional order hyperchaotic system [25]. Lingdong $\mathrm{Z}$ et al. adopted the finite-time stability theory to accomplish the synchronization of hyperchaotic Lorenz systems [26]. In literature [27], a robust non-singular terminal sliding mode controller is proposed for synchronizing two different input nonlinear uncertain chaotic systems. However, these results are aimed at specific chaotic systems. What we need more is a controller that can control all fractional-order chaotic systems. What's more, in real life, chaotic systems do not exist in society in isolation. Of course, there are some disturbances. For example, the signal transmission caused by random fluctuations is a disturbed process. Disturbances can be divided into parameter disturbance [28], external disturbance [29] and internal disturbance [30]. Their presence will make the system unstable and difficult to control. Therefore, some scholars began to consider the impact of disturbance. Literatures [31] [32] [33] have solved the synchronization of fractional-order chaotic systems under random disturbance, but they only considered one of the above three disturbances. If they can consider multiple disturbances, it has more practical significance. Although literatures [34] [35] [36] consider the case of multiple disturbances, the object of their research is the integer order chaotic system.

In response to this situation, we are going to consider the synchronization of fractional-order chaotic systems under two kinds of disturbances, namely para- 
meter disturbances and external disturbances, where these two disturbances are random. The controller we designed has good robustness and anti-interference performance and this method we derived can make the error systems have a faster convergence rate. Many figures provided by the numerical simulations guarantee our theoretical analysis. Meanwhile, conclusions that the synchronised time of finite-time synchronization is shorter and faster than the complete synchronization and the time can be adjusted according to the parameters in the controller are established. The composition of this article is shown below. In chapter 2, we present the definitions, lemmas and stability theories that need to be used. In chapter 3, synchronization conditions are presented. In chapter 4, the numerical simulations proved that our method is very effective. In chapter 5 , we have a sum up for this paper.

\section{Related Theories of Fractional Order System}

\subsection{Definitions and Lemmas of Fractional Derivative}

Next, we will introduce the Riemann-Liouville (R-L) derivative and the Caputo derivative. When the order $\alpha$ is a negative real number and a positive integer, they are equivalent. The R-L definition is more suitable for theoretical analysis and can simplify the calculation of fractional order derivatives. The definition of Caputa is more suitable for modern engineering and makes the Laplace transformation more concise.

Definition 1. [1] The mathematical expression of Caputo derivative with or$\operatorname{der} \alpha$ is given as

$$
{ }_{a}^{C} D_{t}^{\alpha} f(t)=\frac{1}{\Gamma(m-\alpha)} \int_{a}^{t}(t-v)^{m-\alpha-1} f^{(m)}(v) \mathrm{d} v,
$$

where $m-1<\alpha<m, m \in \mathbb{Z}^{+}$.

Definition 2. [1] The mathematical expression of Riemann-Liouville derivative with order $\alpha$ is given as

$$
{ }_{a}^{R L} D_{t}^{\alpha} f(t)=\frac{1}{\Gamma(m-\alpha)} \frac{\mathrm{d}^{m}}{\mathrm{~d} t^{m}} \int_{a}^{t}(t-v)^{m-\alpha-1} f(v) \mathrm{d} v,
$$

where $m-1<\alpha<m, m \in \mathbb{Z}^{+}$.

Lemma 1. [22] When $\boldsymbol{x}(t) \in \mathbb{R}^{n}$ has continuous first derivative, then

$$
{ }_{a} D_{t}^{\alpha}\left(\frac{1}{2} \boldsymbol{x}^{\mathrm{T}}(t) Q \boldsymbol{x}(t)\right) \leq \boldsymbol{x}^{\mathrm{T}} Q_{a} D_{t}^{\alpha} \boldsymbol{x}(t),
$$

where $\alpha \in(0,1)$ and $Q$ is an arbitrary $n$ order positive definite matrix.

\subsection{Stability Theories of Fractional Order System}

Considering that most of the things around us are nonlinear, we write the fractional order nonlinear system to be:

$$
{ }_{0} D_{t}^{a} \boldsymbol{x}(t)=\boldsymbol{f}(t, \boldsymbol{x}(t)),
$$


where $\alpha \in(0,1), \quad \boldsymbol{f}=\left(f_{1}, f_{2}, \cdots, f_{n}\right)^{\mathrm{T}}, \quad \boldsymbol{x}(t) \in \mathbb{R}^{n}$. And $f:\left[t_{0}, \infty\right] \times \Omega \rightarrow \mathbb{R}^{n}$ meets Lipschitz conditions; the initial value is $x\left(t_{0}\right)=x_{0}, t_{0} \geq 0$. The equilibrium point $\boldsymbol{x}^{*}$ of (4) can be obtained from $\boldsymbol{f}\left(\boldsymbol{x}^{*}\right)=0$.

Theorem 1. [37] Suppose that $\mathbb{D} \in \mathbb{R}^{n}$ is a domain that contains the origin. If there is a locally bounded Lyapunov function $V(t, x(t)):\left[t_{0}, \infty\right] \times \mathbb{D} \rightarrow \mathbb{R}$ which meets the local Lipschitz condition about $x$ adapting to

$$
\begin{gathered}
\eta_{1}\left(\|\boldsymbol{x}(t)\|^{a}\right) \leq V(t, \boldsymbol{x}(t)) \leq \eta_{2}\left(\|\boldsymbol{x}(t)\|^{a b}\right), \\
{ }_{0} D_{t}^{\alpha} V(t, \boldsymbol{x}(t)) \leq-\eta_{3}\left(\|\boldsymbol{x}(t)\|^{a b}\right),
\end{gathered}
$$

where $\alpha \in(0,1), a>0, \quad b>0, \eta_{i}(i=1,2,3)>0$, then the system (4) is called Mittag-Leffler stable.

Theorem 2. [37] Suppose that $\mathbb{D} \in \mathbb{R}^{n}$ is a domain that contains the origin. If there is a locally bounded Lyapunov function $V(t, \boldsymbol{x}(t)):\left[t_{0}, \infty\right] \times \mathbb{D} \rightarrow \mathbb{R}$ which meets the local Lipschitz condition about $x$ adapting to

$$
\begin{aligned}
& \text { 1) } \eta_{1}\left(\|\boldsymbol{x}(t)\|^{a}\right) \leq V(t, \boldsymbol{x}(t)) \leq \eta_{2}\left(\|\boldsymbol{x}(t)\|^{a b}\right), \\
& \text { 2) } k V^{1 / \beta}(t, \boldsymbol{x}(t)) \leq \eta_{3}\left(\|\boldsymbol{x}(t)\|^{a b}\right), \\
& \text { 3) }{ }_{0} D_{t}^{\alpha} V(t, \boldsymbol{x}(t)) \leq-\eta_{3}\left(\|\boldsymbol{x}(t)\|^{a b}\right),
\end{aligned}
$$

where $\alpha \in(0,1), a>0, \quad b>0, k>0, \quad \beta>1, \eta_{i}(i=1,2,3)>0$, the system (4) is called finite-time stable. The stabilization time of the system (4) has the following form

$$
T \leq\left(\frac{\beta(\alpha+1)}{k(\beta-1)} V^{(\beta-1) / \beta}\left(0, x_{0}\right)\right)^{1 / \alpha} .
$$

Corollary 1. It follows from Lemma 1 and Theorem 2 that the system (4) must first satisfy the criterions of the Mittag-Leffler stability. From the conditions (2) and (3) in Theorem 2, we get

$$
{ }_{0} D_{t}^{\alpha} V(t, \boldsymbol{x}(t)) \leq-k V^{1 / \beta}(t, \boldsymbol{x}(t)) .
$$

Hence, the system (4) is called finite-time stable if it satisfies (8) and the criterions of the Mittag-Leffler stability.

\section{Sufficients Condition for Finite-Time Synchronization}

In this chapter, we believe that a small disturbance can make a great change in the orbit of the chaotic system. Therefore, it is reasonable to treat them as bounded. This will also make our theory easier to understand. With the help of Lyapulov function, we obtain our conclusions successfully.

The fractional-order drive-response system with parametric disturbance and external disturbance is demonstrated as follows. The drive system is:

$$
{ }_{0} D_{t}^{a} \boldsymbol{x}(t)=(A+\Delta A) \boldsymbol{x}(t)+\boldsymbol{f}(\boldsymbol{x}(t))+\boldsymbol{d}_{1}(\boldsymbol{x}, t) .
$$


The response system is:

$$
{ }_{0} D_{t}^{a} \boldsymbol{y}(t)=(B+\Delta B) \boldsymbol{y}(t)+\boldsymbol{g}(\boldsymbol{y}(t))+\boldsymbol{d}_{2}(\boldsymbol{x}, t)+\boldsymbol{u}(t),
$$

where $A$ and $B$ are the parameter matrices of the systems; $\Delta A$ and $\Delta B$ are the parameter interference matrices; $\boldsymbol{f}(\boldsymbol{x}(t))$ and $\boldsymbol{g}(\boldsymbol{y}(t))$ are the nonlinear vectors; $\boldsymbol{d}_{1}(\boldsymbol{x}, t)$ and $\boldsymbol{d}_{2}(\boldsymbol{x}, t)$ are external disturbances.

We suppose the state error among the driving system with response system as $\boldsymbol{e}(t)=\boldsymbol{y}(t)-\boldsymbol{x}(t)$. Subtract (9) from (10) to get the error system:

$$
{ }_{0} D_{t}^{a} \boldsymbol{e}(t)=C \boldsymbol{y}(t)-D \boldsymbol{x}(t)+\boldsymbol{g}(\boldsymbol{y}(t))-\boldsymbol{f}(\boldsymbol{x}(t))+\boldsymbol{d}(\boldsymbol{x}, t)+\boldsymbol{u}(t),
$$

where $C=B+\Delta B, D=A+\Delta A$ and $\boldsymbol{d}(\boldsymbol{x}, t)=\boldsymbol{d}_{2}(\boldsymbol{x}, t)-\boldsymbol{d}_{1}(\boldsymbol{x}, t)$.

For the sake of ensuring our conclusion more realistic, we need to make the following assumptions.

Assumption 1. For any $\boldsymbol{x}(t) \in \mathbb{R}^{n}$, the nonlinear function $\boldsymbol{g}(\boldsymbol{x}(t))$ and $\boldsymbol{f}(\boldsymbol{x}(t))$ are continuous and smooth. That is, there is a constant $M$ adapting to

$$
\|\boldsymbol{f}(\boldsymbol{x}(t))-\boldsymbol{g}(\boldsymbol{x}(t))\| \leq M,
$$

where $M>0,\|\cdot\|$ represents the 2-norm of matrix.

Assumption 2. For any $\boldsymbol{x}(t), \boldsymbol{y}(t) \in \mathbb{R}^{n}$, the nonlinear function $\boldsymbol{g}(\cdot)$ meets the Lipschitz condition, namely

$$
\|\boldsymbol{g}(\boldsymbol{y}(t))-\boldsymbol{g}(\boldsymbol{x}(t))\| \leq L\|\boldsymbol{y}-\boldsymbol{x}\| .
$$

Remark 1. Regardless of whether the nonlinear functions $\boldsymbol{g}(\cdot)$ and $\boldsymbol{f}(\cdot)$ are about $\boldsymbol{x}$ or $\boldsymbol{y}$, they are bounded because the system state variables $\boldsymbol{x}$ and $\boldsymbol{y}$ are bounded.

Assumption 3. The parameter matrices $A$ and $B$, the parameter interference matrices $\Delta A$ and $\Delta B$, the external disturbances $\boldsymbol{d}_{1}(\boldsymbol{x}, t)$ and $\boldsymbol{d}_{2}(\boldsymbol{x}, t)$ all have a bounded norm, namely

$$
\begin{gathered}
\|\Delta A\|+\|\Delta B\| \leq p, \\
\|A\|+\|B\| \leq q, \\
\left\|\boldsymbol{d}_{2}(\boldsymbol{x}, t)-\boldsymbol{d}_{1}(\boldsymbol{x}, t)\right\| \leq l,
\end{gathered}
$$

where $p>0, q>0, l>0$.

Theorem 3. When the assumptions 1 - 3 are all satisfied, the systems (9) and (10) are stable for a finite time with the following controller:

$$
\boldsymbol{u}(t)=(A+\Delta A) \boldsymbol{y}(t)-(B+\Delta B) \boldsymbol{x}(t)-\left[m_{1} \boldsymbol{e}+\left(m_{2}+1\right) \frac{\boldsymbol{e}}{\|\boldsymbol{e}\|}\right],
$$

where $m_{1} \geq L+p+q, \quad m_{2} \geq l+M$ and $\|\boldsymbol{e}\| \neq 0$.

Proof. Take the Lyapulov function as

$$
V(t, \boldsymbol{x}(t))=\frac{1}{2} \boldsymbol{e}^{\mathrm{T}} \boldsymbol{e} .
$$

The fractional order derivative of the Lyapulov function is 


$$
\begin{aligned}
{ }_{0} & D_{t}^{\alpha} V(t, \boldsymbol{x}(t)) \leq \boldsymbol{e}^{\mathrm{T}}{ }_{0} D_{t}^{\alpha} \boldsymbol{e} \\
= & \boldsymbol{e}^{\mathrm{T}}[(B+\Delta B) \boldsymbol{y}(t)-(A+\Delta A) \boldsymbol{x}(t)+\boldsymbol{g}(\boldsymbol{y}(t))-\boldsymbol{f}(\boldsymbol{x}(t)) \\
& \left.+\boldsymbol{d}_{2}(\boldsymbol{x}, t)-\boldsymbol{d}_{1}(\boldsymbol{x}, t)+\boldsymbol{u}(t)\right] \\
= & \boldsymbol{e}^{\mathrm{T}}[(B \boldsymbol{y}(t)-B \boldsymbol{x}(t))+(A \boldsymbol{y}(t)-A \boldsymbol{x}(t))+(\Delta B \boldsymbol{y}(t)-\Delta B \boldsymbol{x}(t)) \\
& +(\Delta A \boldsymbol{y}(t)-\Delta A \boldsymbol{x}(t))]+(\boldsymbol{g}(\boldsymbol{y}(t))-\boldsymbol{g}(\boldsymbol{x}(t)))+(\boldsymbol{g}(\boldsymbol{x}(t))-\boldsymbol{f}(\boldsymbol{x}(t))) \\
& +\boldsymbol{d}_{2}(\boldsymbol{x}, t)-\boldsymbol{d}_{1}(\boldsymbol{x}, t)-\left[m_{1} \boldsymbol{e}+\left(m_{2}+1\right) \frac{\boldsymbol{e}}{\|\boldsymbol{e}\|}\right] \\
\leq & \|\boldsymbol{e}\|^{2}(\|B\|+\|A\|+\|\Delta B\|+\|\Delta A\|+L)+\|\boldsymbol{e}\| M+l\|\boldsymbol{e}\| \\
& -\|\boldsymbol{e}\| \cdot\left(\left\|m_{1} \boldsymbol{e}+\left(m_{2}+1\right) \frac{\boldsymbol{e}}{\|\boldsymbol{e}\|}\right\|\right) \\
= & \|\boldsymbol{e}\|^{2}(\|B\|+\|A\|+\|\Delta B\|+\|\Delta A\|+L)+\|\boldsymbol{e}\| M+l\|\boldsymbol{e}\|-m_{1}\|\boldsymbol{e}\|^{2}-\left(m_{2}+1\right)\|\boldsymbol{e}\| \\
\leq & \|\boldsymbol{e}\|^{2}(q+p+L)+\|\boldsymbol{e}\| M+l\|\boldsymbol{e}\|-\|\boldsymbol{e}\|^{2}(L+q+p)-\|\boldsymbol{e}\|(l+M+1) \\
= & -\|\boldsymbol{e}\| .
\end{aligned}
$$

Finally, ${ }_{0} D_{t}^{\alpha} V(t, \boldsymbol{x}(t))<0$ is obtained. Therefore, we must be able to find $\alpha_{3}$ to make ${ }_{0} D_{t}^{\alpha} V(t, \boldsymbol{x}(t)) \leq-\alpha_{3}\|x\|^{2}$ hold. It follows from Lemma 2 that the system (4) is Mittag-Leffler stable. Then, because the formula

${ }_{0} D_{t}^{\alpha} V(t, \boldsymbol{x}(t)) \leq-\|\boldsymbol{e}\|=-(2 V(t, \boldsymbol{x}(t)))^{1 / 2}=-\sqrt{2} V(t, \boldsymbol{x}(t))^{1 / 2}$ is held, we can obtain that the systems (9) and (10) achieve finite-time synchronization according to Theorem 1. And

$$
T \leq\left(\frac{2(\alpha+1)}{\sqrt{2}} V^{1 / 2}\left(0, x_{0}\right)\right)^{1 / \alpha},
$$

where $\alpha \in(0,1)$.

\section{Numerical Simulation}

We exemplify a pair of three-dimensional and four-dimensional fractional chaotic systems to affirm the availability of our method. The results indicate that the error variables of the system quickly stabilize in a finite time, and the synchronization time is able to adjust via $m_{1}, m_{2}$. Among them, the influence of $m_{1}$ on the synchronization time is small, and we can ignore it.

Example 1. Let the fractional order Chen chaotic system [38] under stochastic disturbances be the drive system

$$
\begin{aligned}
& { }_{0} D_{t}^{\alpha} x_{1}=(a+\Delta a)\left(x_{2}-x_{1}\right)+d_{11}, \\
& { }_{0} D_{t}^{\alpha} x_{2}=(c+\Delta c-a-\Delta a) x_{1}-x_{1} x_{3}+(c+\Delta c) x_{2}+d_{12}, \\
& { }_{0} D_{t}^{\alpha} x_{3}=x_{2} x_{1}-(b+\Delta b) x_{3}+d_{13},
\end{aligned}
$$

where $\alpha \in(0,1), a=35, b=3, c=28$ and $\boldsymbol{d}_{1}=\left(d_{11}, d_{12}, d_{13}\right)$.

Let the fractional order Lorenz chaotic system [39] under stochastic disturbances and controller be the response system 


$$
\begin{aligned}
& { }_{0} D_{t}^{\alpha} y_{1}=\left(a_{1}+\Delta a_{1}\right)\left(y_{2}-y_{1}\right)+d_{21}+u_{1}, \\
& { }_{0} D_{t}^{\alpha} y_{2}=y_{1}\left(b_{1}+\Delta b_{1}-y_{3}\right)-y_{2}+d_{22}+u_{2}, \\
& { }_{0} D_{t}^{\alpha} y_{3}=y_{2} y_{1}-\left(c_{1}+\Delta c_{1}\right) y_{3}+d_{23}+u_{3},
\end{aligned}
$$

where $\alpha \in(0,1), a_{1}=10, b_{1}=28, c_{1}=8 / 3$ and $\boldsymbol{d}_{2}=\left(d_{21}, d_{22}, d_{23}\right)$.

When we select the initial value as $(1,-2,-2)$ and the order $\alpha=0.995$, the driving system (19) appears chaotic attractors which are presented in Figure 1. For the response system (20), if we take the initial value $(0,1,-1)$ and the order $\alpha=0.995$, it appears chaotic attractors which are presented in Figure 2. We realize that the trajectories about the state variables of fractional order Chen and Lorenz systems are not synchronized with time without any control. Next, we will verify the effectiveness of our controller.

We use the MATLAB software to obtain the state variable trajectory of the systems (19) and (20) obtaining the following results

$$
-21 \leq x_{1} \leq 22,-24 \leq x_{2} \leq 25,7 \leq x_{3} \leq 38,
$$

(a)

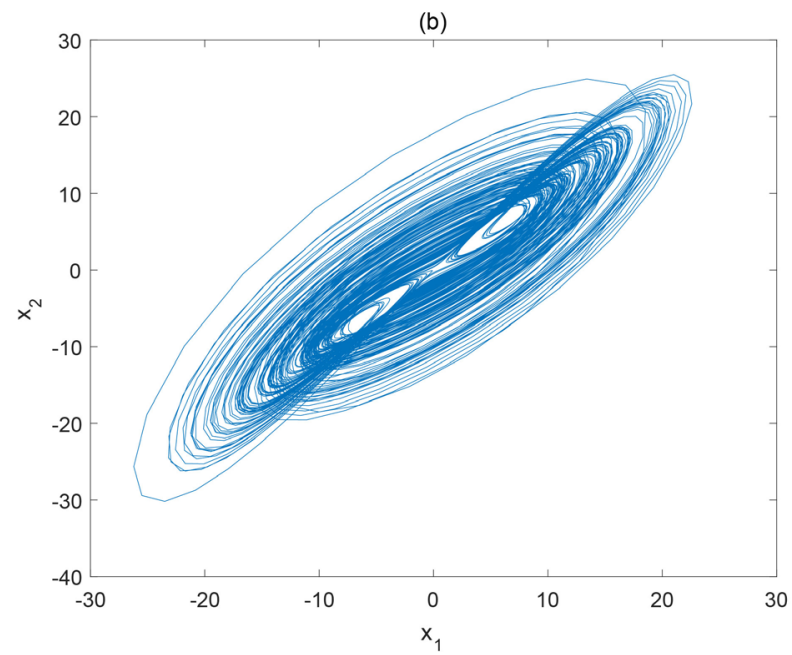

(c)
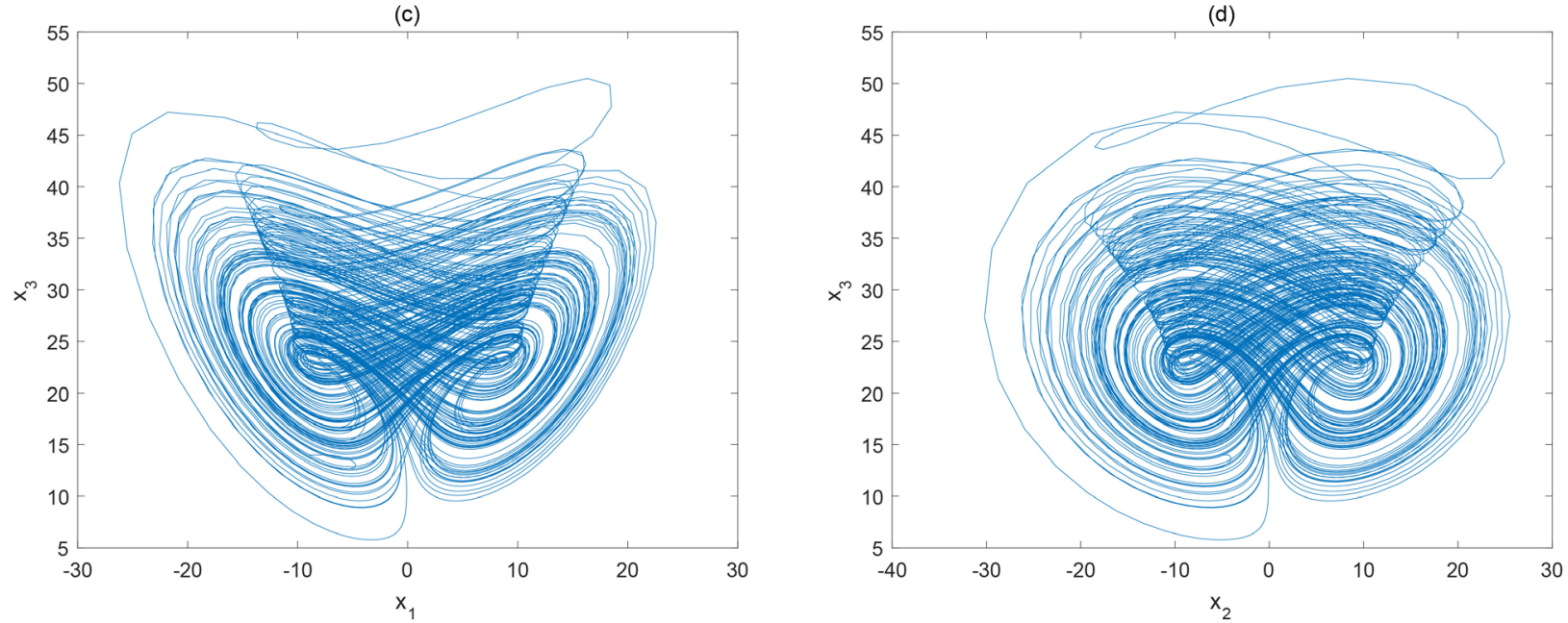

Figure 1. The attractors with respect to fractional order Chen chaotic system choosing $\alpha=0.995$ and $a=35, b=3, c=28$ show in sub-pictures (a)-(d) respectively which are $x_{1}-x_{2}-x_{3}, x_{1}-x_{2}, x_{1}-x_{3}$ and $x_{2}-x_{3}$. 
(a)

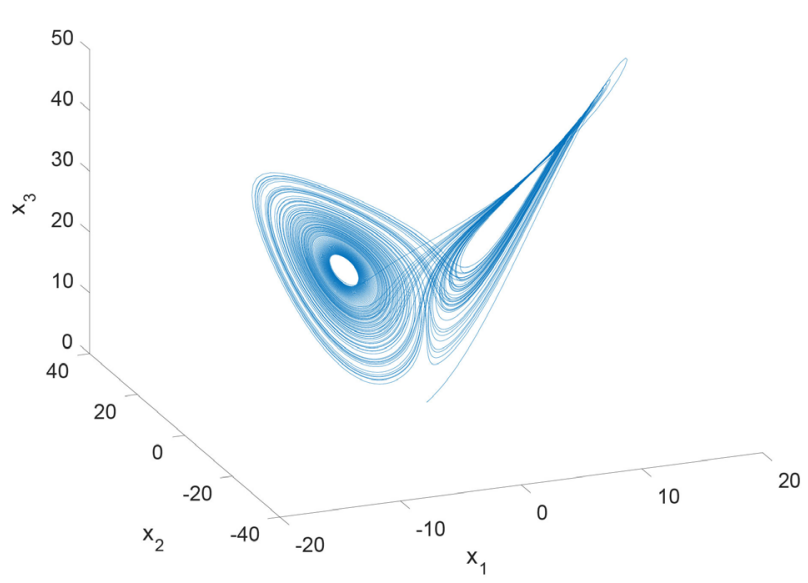

(c)

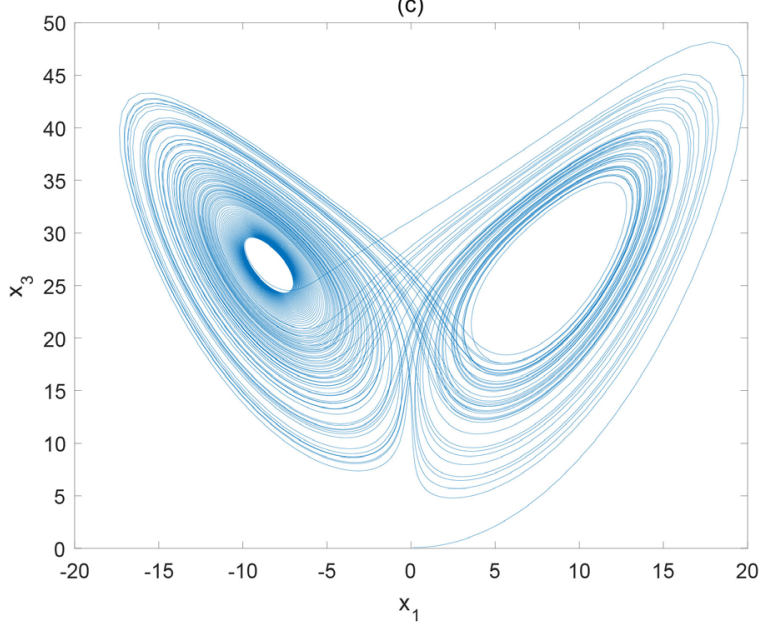

(b)

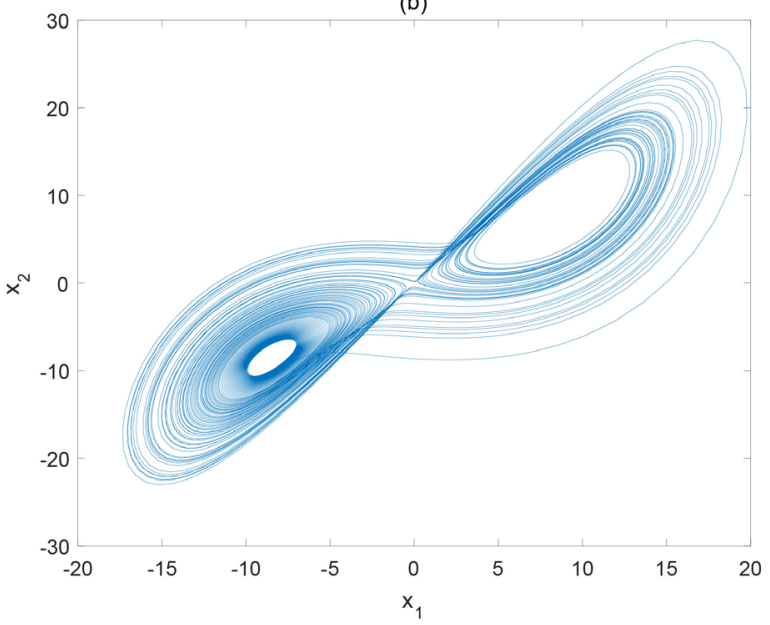

(d)

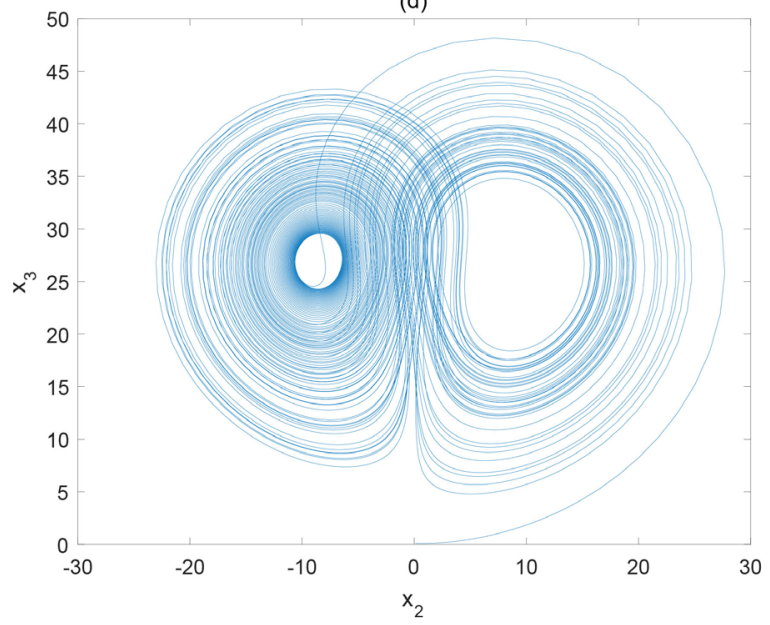

Figure 2. The attractors with respect to fractional order Lorenz chaotic system choosing $\alpha=0.995$ and $a_{1}=10, b_{1}=28, c_{1}=8 / 3$ show in sub-pictures (a)-(d) respectively which are $x_{1}-x_{2}-x_{3}, x_{1}-x_{2}, x_{1}-x_{3}$ and $x_{2}-x_{3}$.

$$
-11 \leq y_{1} \leq 20,-13 \leq y_{2} \leq 28,0 \leq y_{3} \leq 49 \text {. }
$$

According to (19) and (20), we get the following matrices. The parameter matrices are

$$
A=\left(\begin{array}{ccc}
-35 & 35 & 0 \\
-7 & 28 & 0 \\
0 & 0 & -3
\end{array}\right), \quad B=\left(\begin{array}{ccc}
-10 & 10 & 0 \\
28 & -1 & 0 \\
0 & 0 & -8 / 3
\end{array}\right)
$$

The parameter interference matrices are

$$
\begin{aligned}
& \Delta A=\operatorname{diag}(-0.3 \sin t, 0.2 \sin t, 0.15 \sin (3 t)), \\
& \Delta B=\operatorname{diag}(-0.1 \sin t, 0.2 \sin t, 0.3 \sin (3 t)) .
\end{aligned}
$$

The nonlinear vectors of systems are

$$
\boldsymbol{f}(\boldsymbol{x}(t))=\left(\begin{array}{c}
0 \\
-x_{1} x_{3} \\
x_{2} x_{1}
\end{array}\right), \quad \boldsymbol{g}(\boldsymbol{y}(t))=\left(\begin{array}{c}
0 \\
-y_{1} y_{3} \\
y_{2} y_{1}
\end{array}\right) .
$$


The external disturbances are

$$
\boldsymbol{d}_{1}(\boldsymbol{x}, t)=\left(\begin{array}{c}
0.05 x_{2} \sin t \\
0.1 x_{3} \sin (3 t) \\
0.01 x_{1} \cos (2 t)
\end{array}\right), \quad \boldsymbol{d}_{2}(\boldsymbol{x}, t)=\left(\begin{array}{c}
0.6 y_{2} \sin t \\
0.9 y_{3} \sin (3 t) \\
0.8 y_{1} \cos (2 t)
\end{array}\right) .
$$

It follows from the assumptions 1 - 3 and Theorem 3 that the following calculation results are obtained.

$$
\begin{gathered}
\|A\|<58,\|B\|<32,\|\Delta A\|<0.4,\|\Delta B\|<0.4 . \\
\left\|\boldsymbol{d}_{2}(\boldsymbol{x}, t)-\boldsymbol{d}_{1}(\boldsymbol{x}, t)\right\|<46,\|\boldsymbol{f}(\boldsymbol{x}(t))-\boldsymbol{g}(\boldsymbol{x}(t))\|=0, \\
\|\boldsymbol{g}(\boldsymbol{y}(t))-\boldsymbol{g}(\boldsymbol{x}(t))\| \leq 63\|\boldsymbol{y}-\boldsymbol{x}\| .
\end{gathered}
$$

Hence, it follows from Assumption 3 and Theorem 3 that we get

$$
L=63, \quad p=0.8, q=90, l=47, M=0, m_{1} \geq 153.8, \quad m_{2} \geq 47 .
$$

We get the controller as

$$
\boldsymbol{u}(t)=\left(\begin{array}{c}
(-35-0.3 \sin t) y_{1}+35 y_{2}+(10+0.1 \sin t) x_{1}-10 x_{2}-k_{1} \\
-7 y_{1}+(28+0.2 \sin t) y_{2}-28 x_{1}-(0.2 \sin t-1) x_{2}-k_{2} \\
(-3+0.15 \sin (3 t)) y_{3}-(0.3 \sin (3 t)-8 / 3) x_{3}-k_{3}
\end{array}\right) .
$$

where $k_{i}(i=1,2,3)=m_{1}\left(y_{i}-x_{i}\right)-m_{2}\left(y_{i}-x_{i}\right) /\left|y_{i}-x_{i}\right|$.

Under the control of the above controller, the synchronised time satisfies $T \leq 4.21$ seconds. Numerical simulations show that the (19) and (20) achieve the finite-time synchronization revealing in Figures 3-5. Comparing Figures $3-5$, it's worth noting that the synchronization time gradually decreases as the

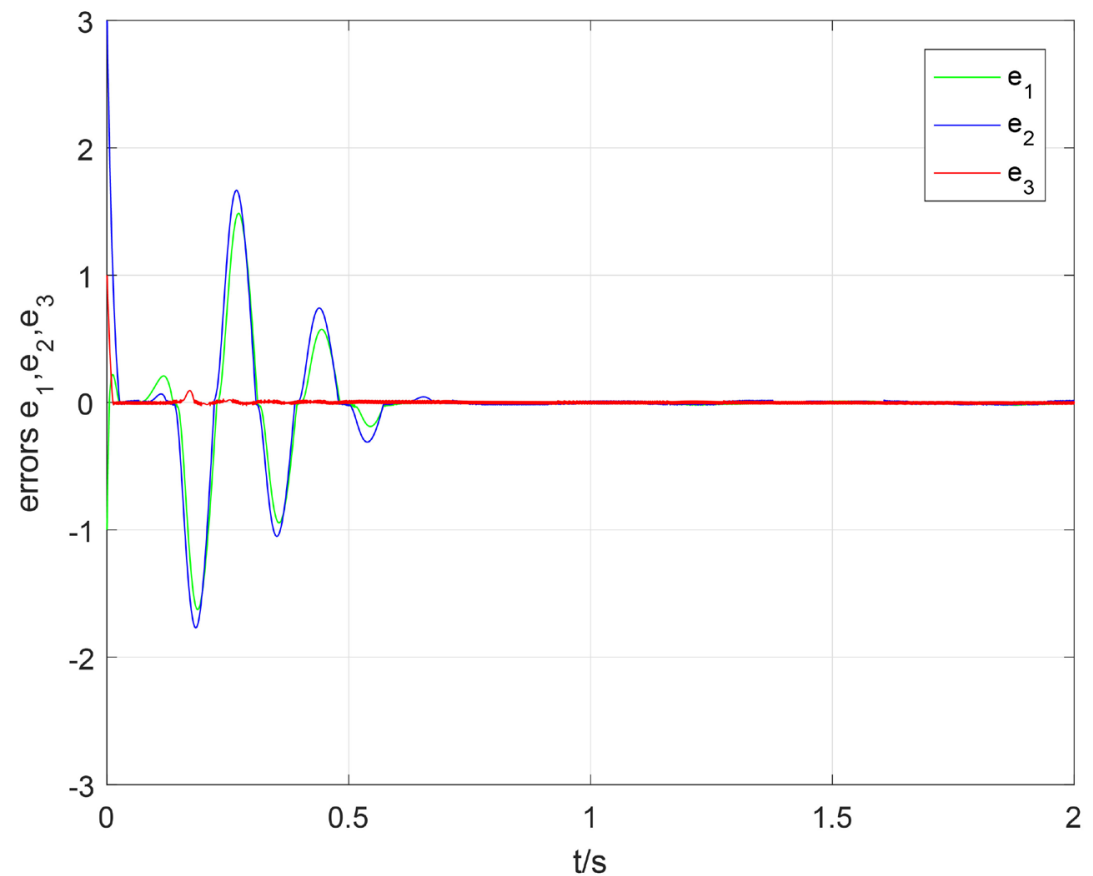

Figure 3. The finite-time synchronization errors $e_{1}, e_{2}, e_{3}$ change with time $t$ when $m_{1}=154, m_{2}=55$. The synchronization time is 0.73 second. 


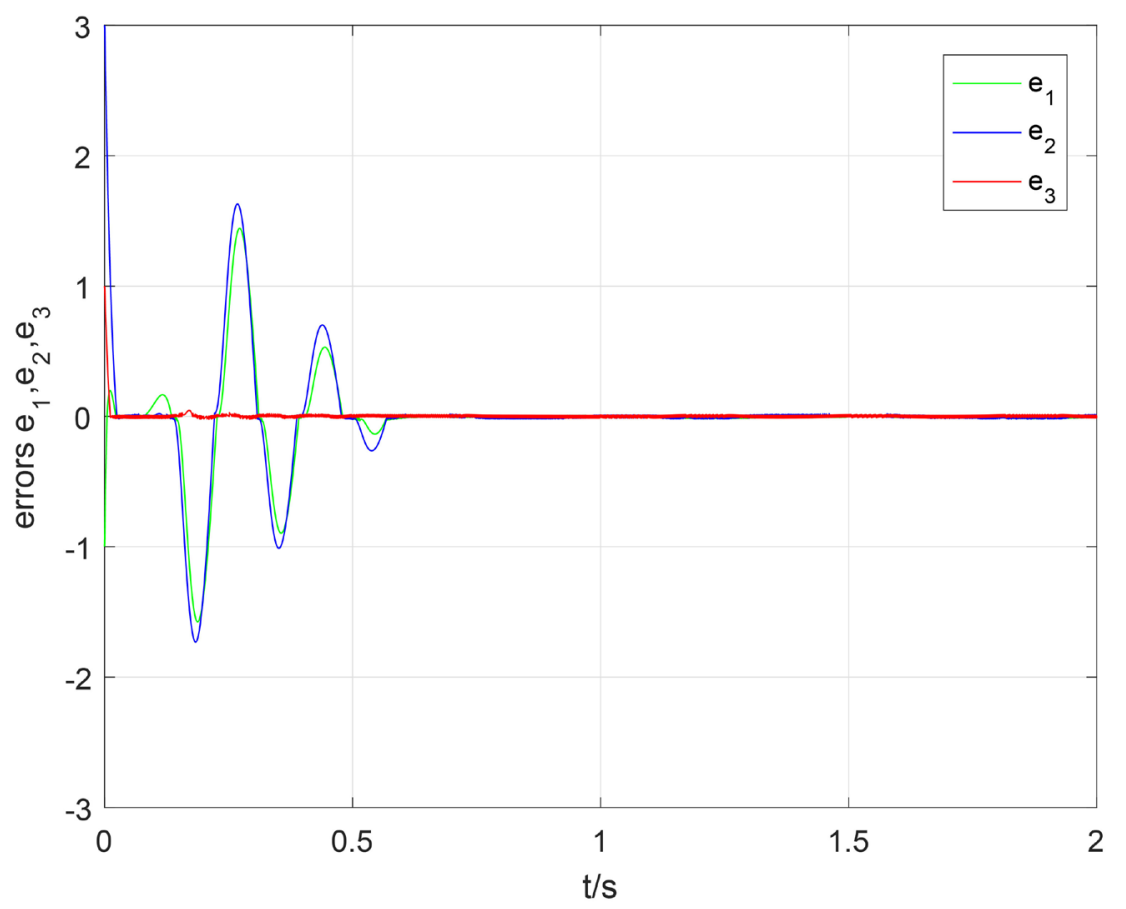

Figure 4. The finite-time synchronization errors $e_{1}, e_{2}, e_{3}$ change with time $t$ when $m_{1}=154, m_{2}=64$. The synchronization time is 0.58 second.

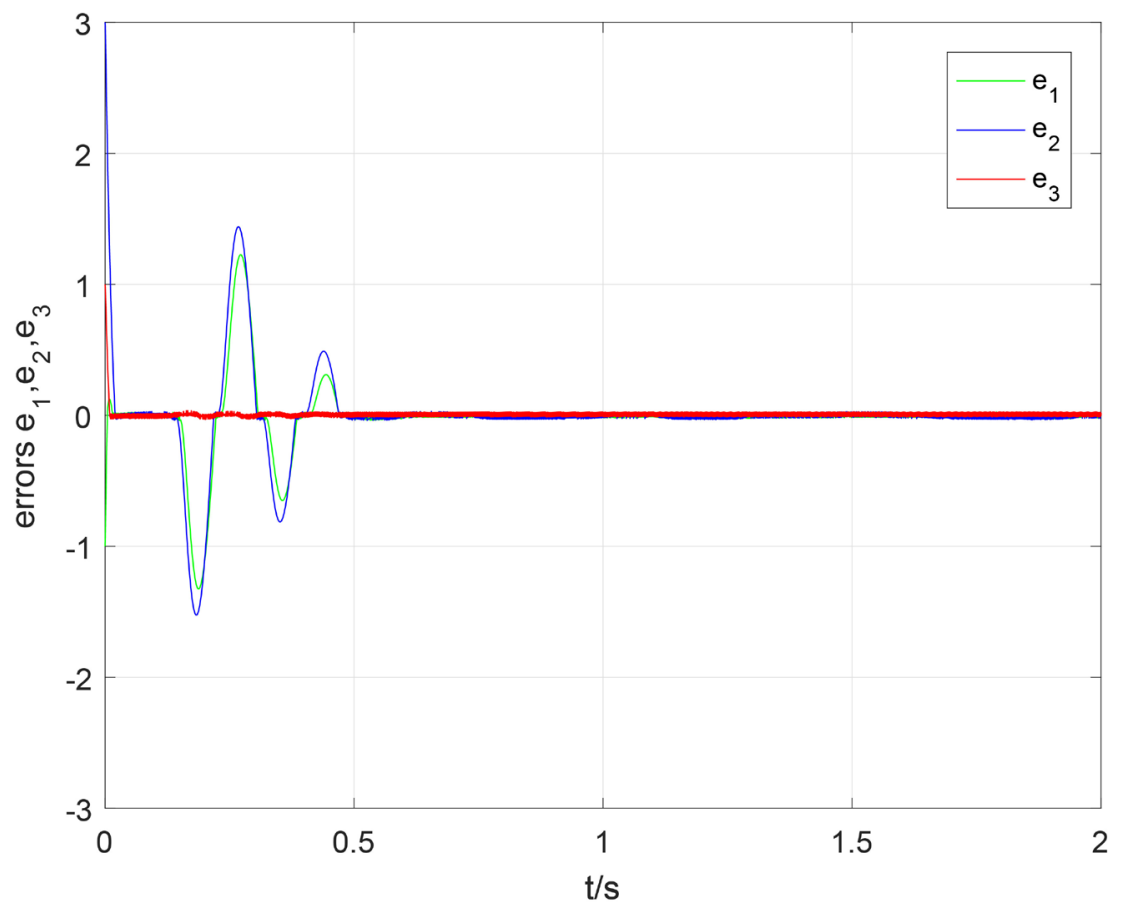

Figure 5. The finite-time synchronization errors $e_{1}, e_{2}, e_{3}$ change with time $t$ when $m_{1}=154, m_{2}=100$. The synchronization time is 0.46 second.

value of $m_{2}$ increases. We can also say that the synchronised time of finite-time synchronization is shorter and faster than the complete synchronization in infi- 
nite time, and it can be adjusted according to the $m_{2}$.

Example 2. Let the fractional order hyperchaotic Lorenz system [38] under stochastic disturbances be the driving system

$$
\begin{aligned}
& { }_{0} D_{t}^{\alpha} x_{1}=(a+\Delta a)\left(x_{2}-x_{1}\right)+x_{4}+d_{11}, \\
& { }_{0} D_{t}^{\alpha} x_{2}=(c+\Delta c) x_{1}-x_{1} x_{3}-x_{2}+d_{12}, \\
& { }_{0} D_{t}^{\alpha} x_{3}=x_{2} x_{1}-(b+\Delta b) x_{3}+d_{13}, \\
& { }_{0} D_{t}^{\alpha} x_{4}=-x_{2} x_{3}+(d+\Delta d) x_{4}+d_{14} .
\end{aligned}
$$

where $\alpha \in(0,1), \quad a=10, b=8 / 3, c=-28, d=-1$ and $\boldsymbol{d}_{1}=\left(d_{11}, d_{12}, d_{13}, d_{14}\right)$.

Let the fractional order hyperchaotic Liu chaotic system [40] under stochastic disturbances and controller be the response system

$$
\begin{aligned}
& { }_{0} D_{t}^{\alpha} y_{1}=\left(a_{1}+\Delta a_{1}\right)\left(y_{2}-y_{1}\right)+d_{21}+u_{1}, \\
& { }_{0} D_{t}^{\alpha} y_{2}=-y_{1} y_{3}+\left(b_{1}+\Delta b_{1}\right) y_{1}+y_{4}+d_{22}+u_{2}, \\
& { }_{0} D_{t}^{\alpha} y_{3}=(m+\Delta m) y_{1}^{2}-\left(c_{1}+\Delta c_{1}\right) y_{3}+d_{23}+u_{3}, \\
& { }_{0} D_{t}^{\alpha} y_{4}=-\left(d_{1}+\Delta d_{1}\right) y_{1}+d_{24}+u_{4} .
\end{aligned}
$$

where $\alpha \in(0,1), a_{1}=10, b_{1}=40, c_{1}=2.5, d_{1}=10, m=4$ and $\boldsymbol{d}_{2}=\left(d_{21}, d_{22}, d_{23}, d_{24}\right)$.

When we select the initial value as $(11,2,1,-1)$ and the order $\alpha=0.99$, the driving system (26) appears chaotic attractors which are presented in Figure 6. For the response system (27), if we take the initial value $(3,-4,2,1)$ and the order $\alpha=0.99$, it appears chaotic attractors which are presented in Figure 7. We realize that the trajectories about the state variables of fractional order hyperchaotic Lorenz and Liu systems are not synchronized with time without any control. Next, we will verify the effectiveness of our controller.

We use the MATLAB software to obtain the state variable trajectory of the systems (26) and (27) obtaining the following results

$$
\begin{aligned}
& -20 \leq x_{1} \leq 25, \quad-25 \leq x_{2} \leq 28, \quad 0 \leq x_{3} \leq 47, \quad-124 \leq x_{4} \leq 180, \\
& -15 \leq y_{1} \leq 20, \quad-35 \leq y_{2} \leq 30, \quad 0 \leq y_{3} \leq 110, \quad-40 \leq y_{4} \leq 40 .
\end{aligned}
$$

According to (26) and (27), we get the following matrices. The parameter matrices are

$$
A=\left(\begin{array}{cccc}
-10 & 10 & 0 & 1 \\
-28 & -1 & 0 & 0 \\
0 & 0 & -8 / 3 & 0 \\
0 & 0 & 0 & -1
\end{array}\right), \quad B=\left(\begin{array}{cccc}
-10 & 10 & 0 & 0 \\
40 & 0 & 0 & 1 \\
0 & 0 & -2.5 & 0 \\
-10 & 0 & 0 & 0
\end{array}\right)
$$

The parameter interference matrices are

$$
\begin{aligned}
& \Delta A=\operatorname{diag}(-0.1 \sin t, 0.2 \sin (2 t), 0.3 \sin (3 t), 0.4 \sin (4 t)), \\
& \Delta B=\operatorname{diag}(-0.1 \cos t, 0.2 \cos (2 t), 0.3 \cos (3 t), 0.4 \cos (4 t)) .
\end{aligned}
$$

The nonlinear vectors of systems are 
(a)

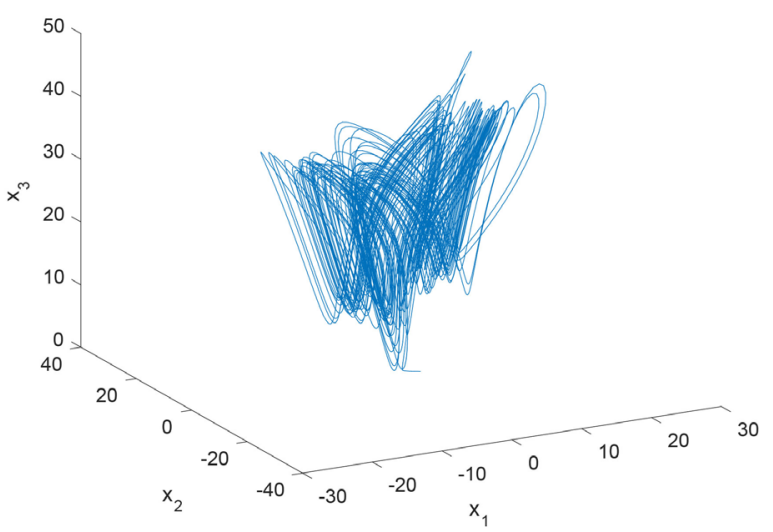

(c)

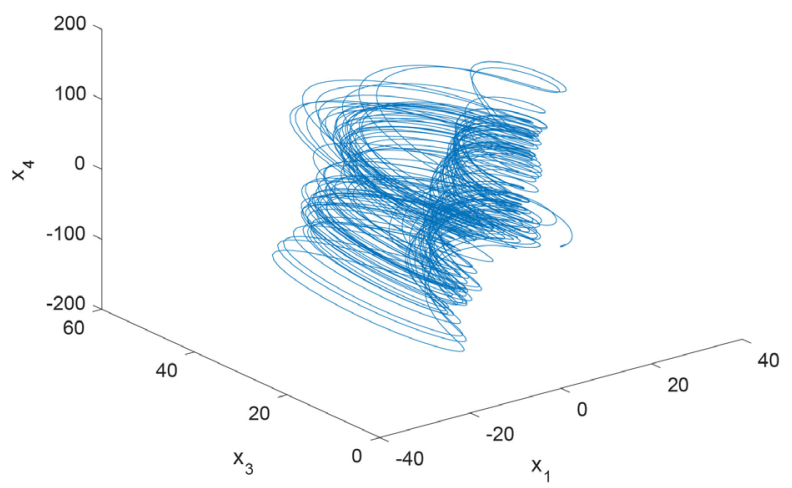

(b)

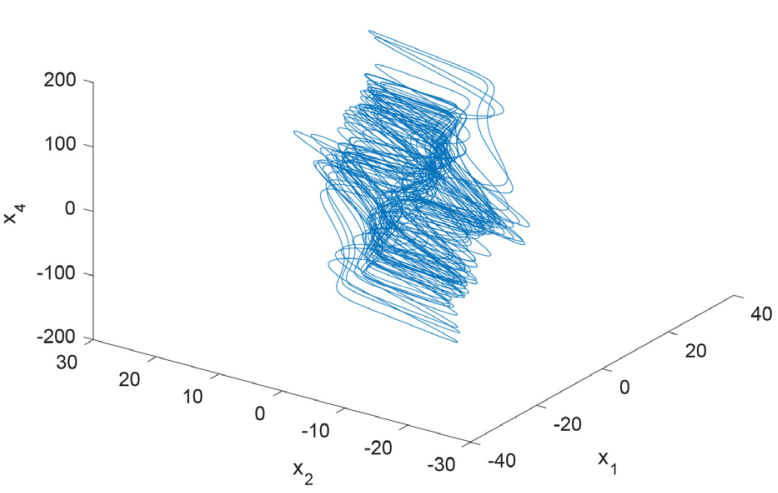

(d)

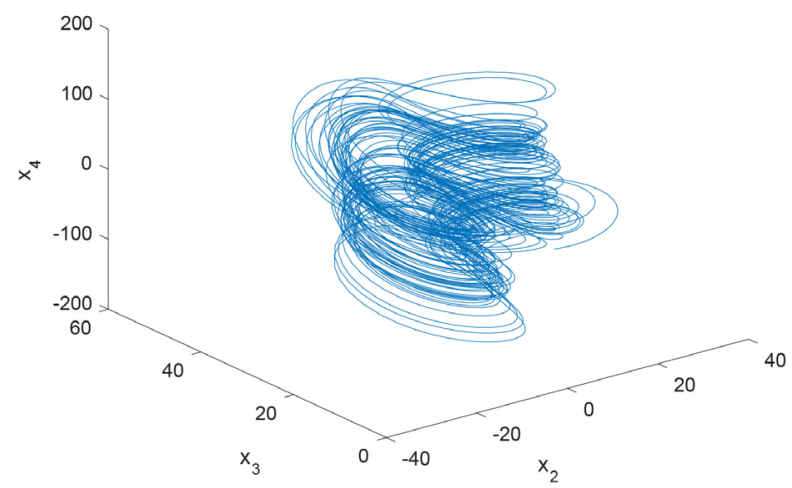

Figure 6. The attractors with respect to fractional order hyper-chaotic Lorenz system choosing $\alpha=0.99$ and $a=10, b=8 / 3, c=28, d=-1$ show in sub-pictures (a)-(d) respectively which are $x_{1}-x_{2}-x_{3}, x_{1}-x_{2}-x_{4}, x_{1}-x_{3}-x_{4}$ and $x_{2}-x_{3}-x_{4}$.

$$
\boldsymbol{f}(\boldsymbol{x}(t))=\left(\begin{array}{c}
0 \\
-x_{1} x_{3} \\
x_{2} x_{1} \\
-x_{2} x_{3}
\end{array}\right), \quad \boldsymbol{g}(\boldsymbol{y}(t))=\left(\begin{array}{c}
0 \\
-y_{1} y_{3} \\
4 y_{1}^{2} \\
0
\end{array}\right) .
$$

The external disturbances are

$$
\boldsymbol{d}_{1}(\boldsymbol{x}, t)=\left(\begin{array}{l}
-0.1 \cos t \\
-0.1 \cos t \\
-0.1 \cos t \\
-0.1 \cos t
\end{array}\right), \quad \boldsymbol{d}_{2}(\boldsymbol{x}, t)=\left(\begin{array}{l}
0.1 \cos t \\
0.1 \cos t \\
0.1 \cos t \\
0.1 \cos t
\end{array}\right) .
$$

It follows from the assumptions $1-3$ and Theorem 3 that the following calculation results are obtained.

$$
\begin{gathered}
\|A\|<32,\|B\|<44,\|\Delta A\|<0.55,\|\Delta B\|<0.55 . \\
\left\|\boldsymbol{d}_{2}(\boldsymbol{x}, t)-\boldsymbol{d}_{1}(\boldsymbol{x}, t)\right\|<0.4,\|f(\boldsymbol{x}(t))-g(\boldsymbol{x}(t))\|<3004, \\
\|g(\boldsymbol{y}(t))-g(\boldsymbol{x}(t))\| \leq 213\|\boldsymbol{y}-\boldsymbol{x}\| .
\end{gathered}
$$


(a)

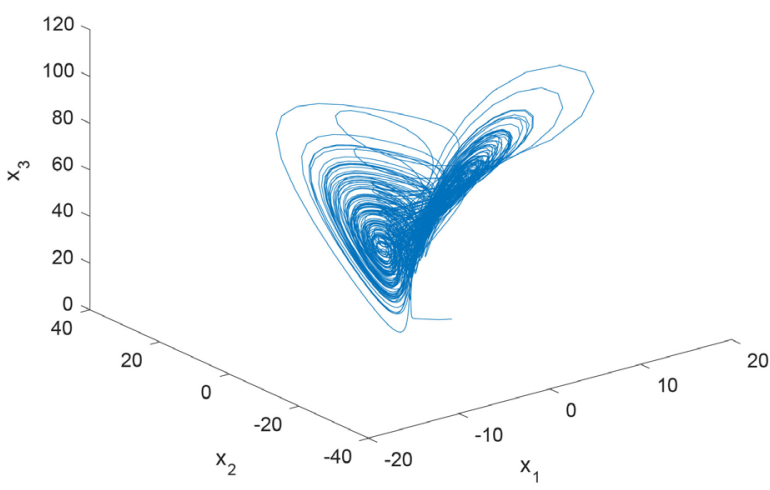

(c)

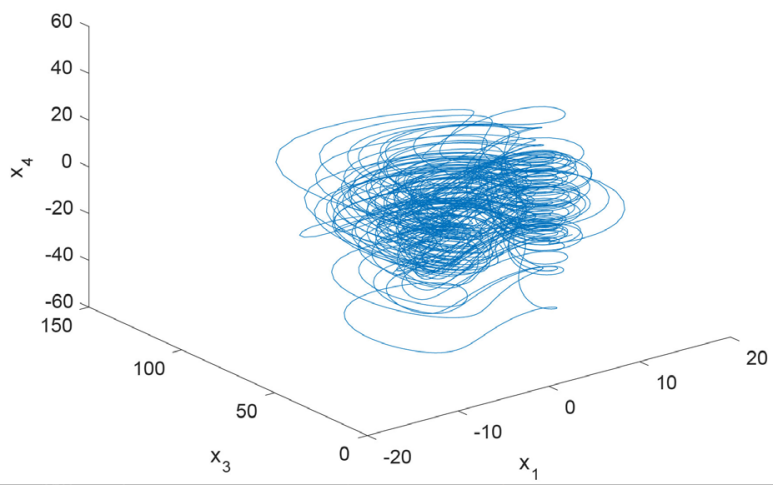

(b)

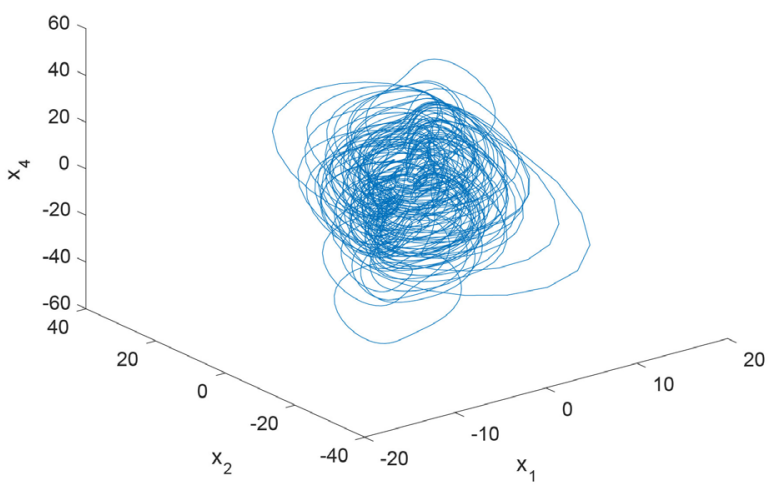

(d)

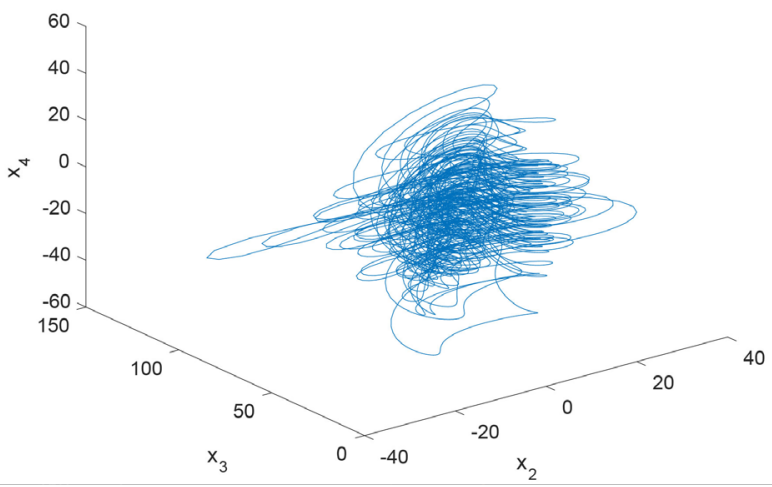

Figure 7. The attractors with respect to fractional order hyperchaotic Liu system choosing $\alpha=0.99$ and $a_{1}=10, b_{1}=40, c_{1}=2.5, m=4, d_{1}=10$ show in sub-pictures (a)-(d) respectively which are $x_{1}-x_{2}-x_{3}, x_{1}-x_{2}-x_{4}, x_{1}-x_{3}-x_{4}$ and $x_{2}-x_{3}-x_{4}$.

Hence, it follows from Assumption 3 and Theorem 3 that we get

$$
L=213, p=1.2, q=76, l=0.4, M=3004, m_{1} \geq 290.2, m_{2} \geq 3004.4 \text {. }
$$

We get the controller as

$$
\boldsymbol{u}(t)=\left(\begin{array}{c}
(-10-0.1 \sin t) y_{1}+10 y_{2}+y_{4}+(10+0.1 \cos t) x_{1}-10 x_{2}-k_{1} \\
-28 y_{1}+(-1+0.2 \sin (2 t)) y_{2}-40 x_{1}-(0.2 \cos (2 t)) x_{2}-x_{4}-k_{2} \\
(-8 / 3+0.3 \sin (3 t)) y_{3}-(-2.5+0.3 \cos (3 t)) x_{3}-k_{3} \\
(-1+0.4 \sin (4 t)) y_{4}+10 x_{1}-(0.4 \cos (4 t)) x_{4}-k_{4}
\end{array}\right) .
$$

where $k_{i}(i=1,2,3,4)=m_{1}\left(y_{i}-x_{i}\right)-m_{2}\left(y_{i}-x_{i}\right) /\left|y_{i}-x_{i}\right|$.

Under the control of above controller, the synchronised time satisfies $T \leq 6.47$ seconds. Numerical simulations show that the (26) and (27) achieve the finite-time synchronization revealing in Figures 8-10. Comparing Figures 8-10, it's worth noting that the synchronization time gradually decreases as the value of $m_{2}$ increases. We can also say that the synchronised time of finite-time synchronization is shorter and faster than the complete synchronization in infinite time, and it can be adjusted according to the $m_{2}$. 


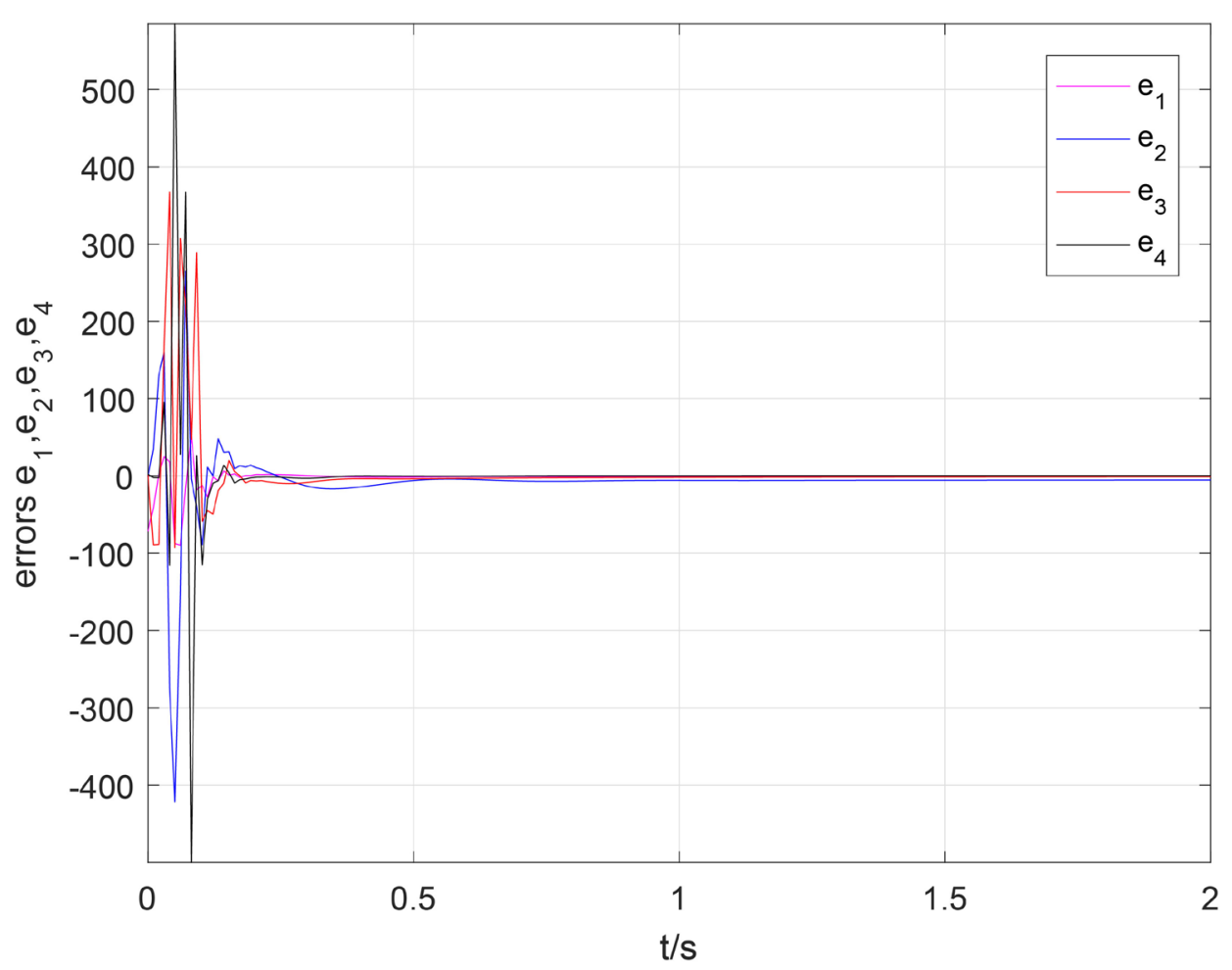

Figure 8. The finite-time synchronization errors $e_{1}, e_{2}, e_{3}, e_{4}$ change with time $t$ when $m_{1}=291, m_{2}=3005$. The synchronization time is 0.50 second.

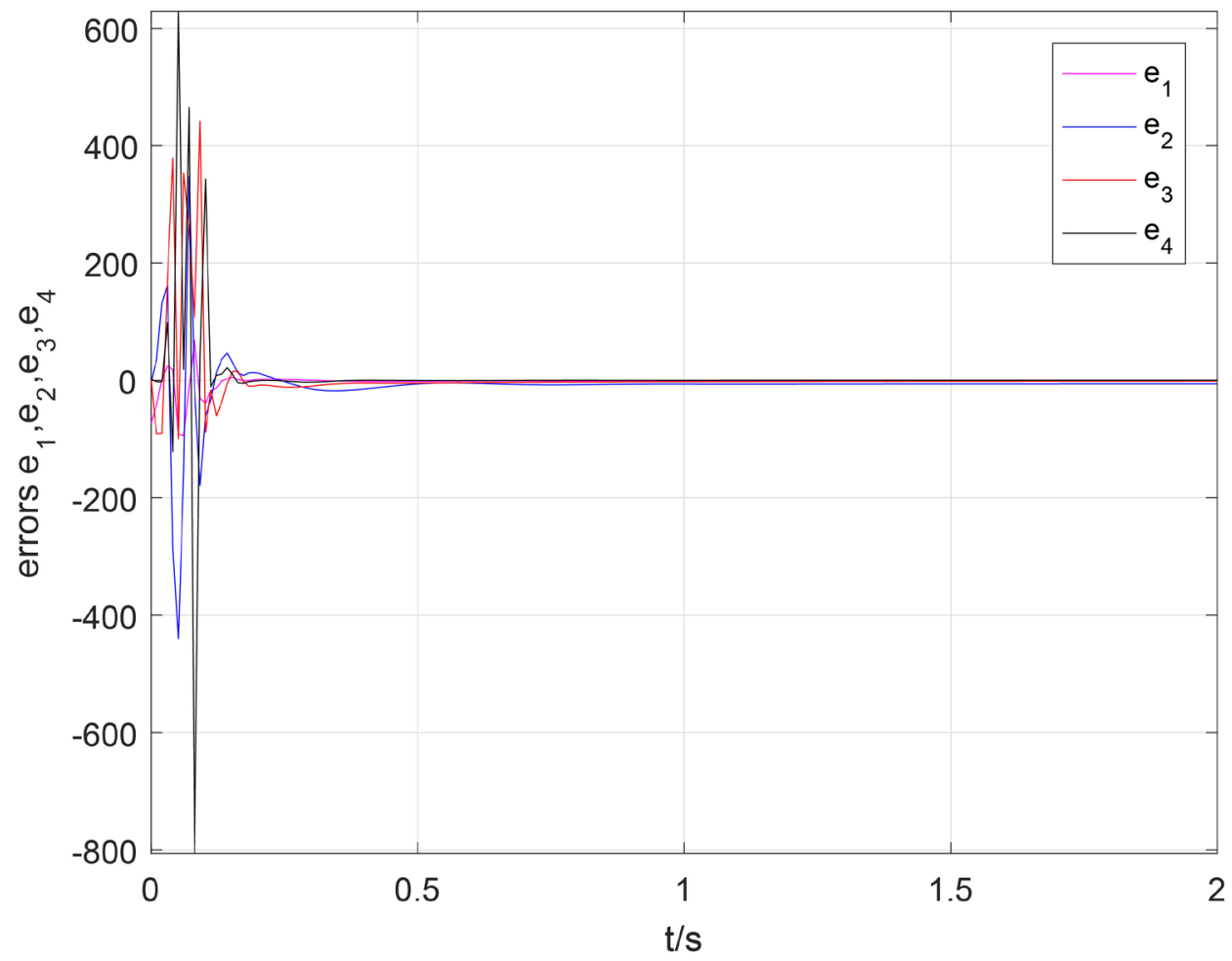

Figure 9. The finite-time synchronization errors $e_{1}, e_{2}, e_{3}, e_{4}$ change with time $t$ when $m_{1}=291, m_{2}=3025$. The synchronization time is 0.45 second. 


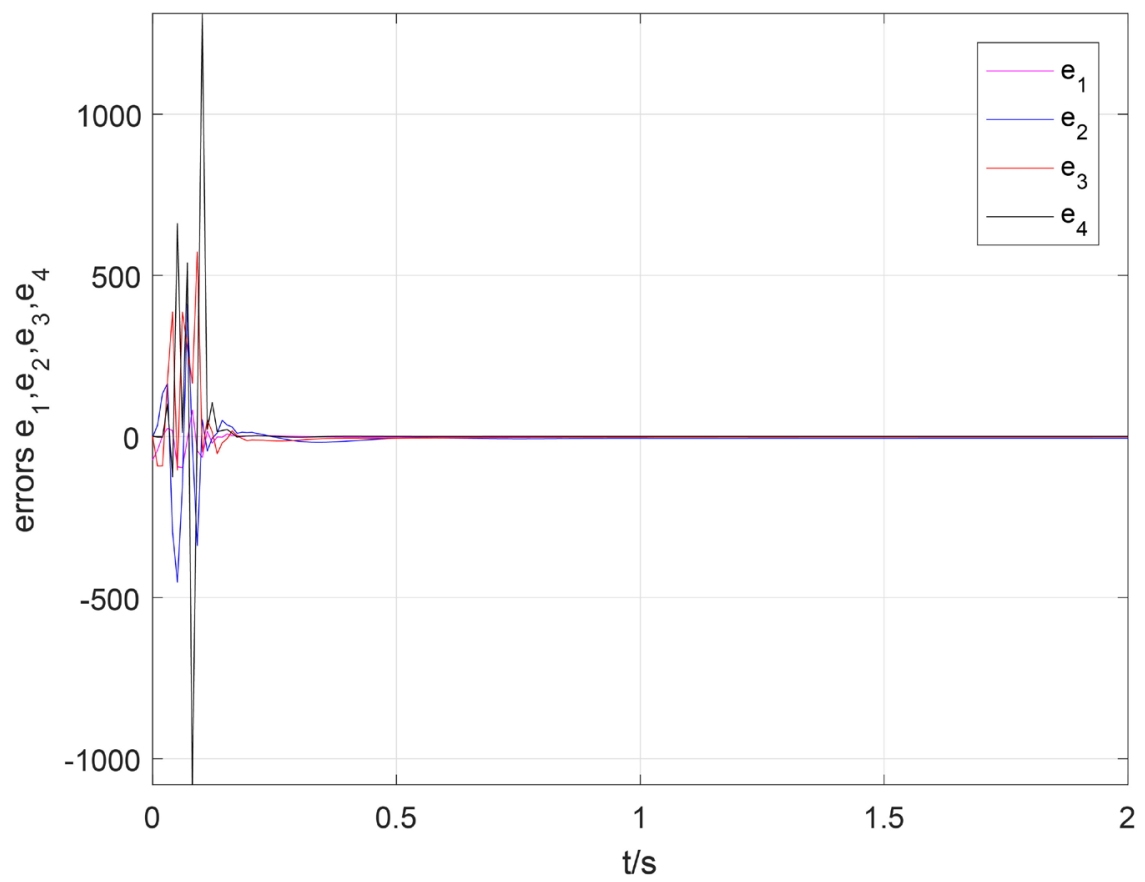

Figure 10. The finite-time synchronization errors $e_{1}, e_{2}, e_{3}, e_{4}$ change with time $t$ when $m_{1}=291, m_{2}=3065$. The synchronization time is 0.27 second.

\section{Conclusion}

In this article, we have studied the finite-time synchronization of fractional-order chaotic systems with different structures under parameter disturbance and external disturbance. With the help of theory of fractional order calculus and the finite-time Lyapunov principle, we put forward a new fractional-order controller which can synchronize any-order fractional-order chaotic system under stochastic disturbances. From the numerical simulation results, it can be seen that the error variables of the systems quickly converges to the equilibrium point in a finite time and the synchronization time gradually decreases with the increase of $m_{2}$. Compared with complete synchronization in infinite time, the synchronised time of finite-time synchronization is shorter and faster and the time can be adjusted according to the parameter value in controller. Therefore, this controller is effective and has strong robustness. Next, we will study the time-delay systems under stochastic disturbances, and the system parameters are unknown.

\section{Acknowledgements}

Here, I would like to thank my instructor, professor Li Tianzeng. It was Teacher Li's careful guidance that allowed me to successfully complete the research work.

\section{Funding}

This work was supported by College Student Innovation and Entrepreneurship Training Program of Sichuan University of Science and Engineering [grant 
numbers cx2020189].

\section{Authors Contributions}

WQ PAN proposed the main the idea and prepared the manuscript initially. TZ LI gave the numerical simulation of this paper.

\section{Availability of Data and Material}

The data used to support the findings of this study are available from the corresponding author upon request.

\section{Conflicts of Interest}

The authors declare that they have no competing interests.

\section{References}

[1] Podlubny, I. (1999) Fractional Differential Equations. Academic, New York.

[2] Hilfer, R. (2001) Applications of Fractional Calculus in Physics. World Scientific, Singapore. https://doi.org/10.1142/3779

[3] Bagley, R.L. (1998) On the Fractional Calculus Model of Viscoelastic Behavior. Journal of Rheology, 30, 133-155. https://doi.org/10.1122/1.549887

[4] Ge, Z.M. and Jhuang, W.R. (2007) Chaos, Control and Synchronization of a Fractional Order Rotational Mechanical System with a Centrifugal Governor. Chaos Solitons \& Fractals, 33, 270-289. https://doi.org/10.1016/j.chaos.2005.12.040

[5] Huang, L.L. and He, S.J. (2011) Stability of Fractional State Space System and Its Application to Fractional Order Chaotic System. Acta Physica Sinica, 60, 353-358. https://doi.org/10.7498/aps.60.044703

[6] Hudson, J.L., Kube, M. and Adomaitis, R.A. (1990) Nonlinear Signal Processing and System Identification, Applications to Time Series from Electrochemical Reactions. Chemical Engineering Science, 45, 2075-2081. https://doi.org/10.1016/0009-2509(90)80079-T

[7] Argenti, F., Angeli, A.D. and Re, E.D. (1997) Secure Communications Based on Discrete Time Chaotic Systems. Kybernetika, 33, 41-50.

[8] Gerhards, M. and Schlerf, M.R. (2018) Analysis of Airborne Optical and Thermal Imagery for Detection of Water Stress Symptoms. Remote Sensing, 10, 1139. https://doi.org/10.3390/rs10071139

[9] Lei, Y. and Zheng, W. (2015) Research on Robot Automation and Control Problems. The World of Inverters, 3, 86-88.

[10] Yang, L.L. and Rong, Z. (1998) An Improvement and Proof of OGY Method. Applied Mathematics \& Mechanics, 1, 1-7. https://doi.org/10.1007/BF02458974

[11] Ping, Z. and Peng, Z. (2002) Drive-Response Synchronization for Chaotic Systems. Journal of Chongqing University, 25, 77-79.

[12] Porfiri, M. and Fiorilli, F. (2009) Global Pulse Synchronization of Chaotic Oscillators through Fast-Switching Theory and Experiments. Chaos Solitons \& Fractals, 41, 245-262. https://doi.org/10.1016/j.chaos.2007.11.033

[13] Yan, J.J., Hung, M.L. and Chiang, T.Y. (2006) Robust Synchronization of Chaotic Systems via Adaptive Sliding Mode Control. Physics Letters A, 356, 220-225. https://doi.org/10.1016/j.physleta.2006.03.047 
[14] Agrawal, S.K., Srivastava, M. and Das, S. (2012) Synchronization of Fractional Order Chaotic Systems Using Active Control Method. Chaos Solitons \& Fractals, 45, 737-752. https://doi.org/10.1016/j.chaos.2012.02.004

[15] Li, R. and Chen, W. (2014) Lyapunov-Based Fractional-Order Controller Design to Synchronize a Class of Fractional-Order Chaotic Systems. Nonlinear Dynamics, 76, 785-795. https://doi.org/10.1007/s11071-013-1169-0

[16] Tao, C. and Liu, X. (2007) Feedback and Adaptive Control and Synchronization of a Set of Chaotic and Hyperchaotic Systems. Chaos Solitons \& Fractals, 32, 1572-1581. https://doi.org/10.1016/j.chaos.2005.12.005

[17] Xi, X.J., Mobayen, S. and Ren, H.P. (2018) Robust Finite-Time Synchronization of a Class of Chaotic Systems via Adaptive Global Sliding Mode Control. Journal of Vibration and Control, 24, 3842-3854. https://doi.org/10.1177/1077546317713532

[18] Li, X. and Zhao, X.S. (2020) The Chaotic Synchronization of Fractional-Order and Integer-Order in a Class of Financial Systems. Journal of Science of Teachers' College and University, 40, 1-4.

[19] Jing, W. and Guang, P. (2016) Design of a Sliding Mode Controller for Synchronization of Fractional-Order Chaotic Systems with Different Structures. Journal of Shanghai Jiaotong University, 50, 849-853+860.

[20] Jiang, N. (2014) The Adaptive Control Synchronization of Hyperchaos Lorenz System and Hyperchaos Rössler System. Journal of Taiyuan Normal University (Natural Science Edition), 13, 47-50.

[21] Wei, X. (2015) Adaptive Control and Synchronization of Lü Hyperchaotic System. Journal of Honghe University, 13, 23-27.

[22] Li, T., Wang, Y. and Zhao, C. (2017) Synchronization of Fractional Chaotic Systems Based on a Simple Lyapunov Function. Advances in Difference Equations, 2007, 304. https://doi.org/10.1186/s13662-017-1320-1

[23] Wei, Y.H. and Chen, Y.Q. (2019) Lyapunov Functions for Nabla Discrete Fractional Order Systems. ISA Transactions, 88, 82-90. https://doi.org/10.1016/j.isatra.2018.12.016

[24] Bhat, S.P. and Bernstein, D.S. (1998) Continuous Finite-Time Stabilization of the Translational and Rotational Double Integrators. IEEE Transactions on Automatic Control, 43, 678-682. https://doi.org/10.1109/9.668834

[25] Sao, K.Y. and Feng, H. (2018) Finite-Time Synchronization of a Class of Fractional-Order Hyperchaotic System. Control and Instruments in Chemical Industry, 45, $1-4+22$.

[26] Zhao, L.D., Hu, J.B. and Bao, Z.H. (2011) A Finite-Time Stable Theorem about Fractional Systems and Finite-Time Synchronizing Fractional Super Chaotic Lorenz Systems. Acta Physica Sinica, 60, 100-104. https://doi.org/10.7498/aps.60.100507

[27] Aghababa, M.P. and Aghababa, P.H. (2013) A Novel Finite-Time Sliding Mode Controller for Synchronization of Chaotic Systems with Input Nonlinearity. Arabian Journal for Science \& Engineering, 38, 3221-3232. https://doi.org/10.1007/s13369-012-0459-Z

[28] Abdurahman, K. and Wang, X.Y. (2011) Adaptive Synchronization for Hyperchaotic Systems with Disturbance of Parameter. Computer Engineering \& Applications, $47,1-3+24$.

[29] Wang, J., Tu, L.L. and Ze, Z.F. (2017) Finite-Time Synchronization for Unified Chaotic System with Stochastic Perturbation. Journal of Mathematics, 37, 193-200.

[30] Xu, Y.H., Xie, C.R., et al. (2014) On Dynamic Analysis of Chaotic Finance System 
with Perturbation. Journal of Yangtze University (Natural Science Edition), 11, $1-4+9-10$.

[31] Gao, Y., Sun, B. and Lu, G. (2013) Modified Function Projective Lag Synchronization of Chaotic Systems with Disturbance Estimations. Applied Mathematical Modelling, 37, 4993-5000. https://doi.org/10.1016/j.apm.2012.09.058

[32] Shao, K.Y., Han, F. and Guo, H.X. (2018) Adaptive Finite Time Control for a Class of Fractional Hyperchaotic Systems. Journal of Jilin University (Information Science Edition), 36, 34-40.

[33] Mao, B.X. (2020) Finite Time Synchronization of Fractional-Order Uncertain LIKE-Bao System. Journal of Jilin University (Science Edition), 58, 145-150.

[34] Wu, J., Ma, Z.C. and Sun, Y.Z. (2015) Finite-Time Synchronization of Chaotic Systems with Noise Perturbation. Kybernetika, 54, 137-149.

https://doi.org/10.14736/kyb-2015-1-0137

[35] Lin, M.L., Yuan, Z.Z. and Cai, J.P. (2019) Finite-Time Synchronization between Two Different Chaotic Systems with Uncertainties. Journal of Fujian University of Technology, 17, 80-85+91.

[36] Lan, T.L. and Wang, Y.J. (2018) Finite-Time Synchronization and Parameters Identification of a Uncertain Critical Chaotic System. Mathematics in Practice and Theory, 48, 105-112.

[37] Li, Y., Chen, Y.Q. and Podlubny, I. (2009) Stability of Fractional-Order Nonlinear Dynamic Systems: Lyapunov Direct Method and Generalized Mittag-Leffler Stability. Computers \& Mathematics with Applications, 59, 1810-1821. https://doi.org/10.1016/j.camwa.2009.08.019

[38] Petras, I. (2011) Fractional-Order Nonlinear Systems, Modeling, Analysis and Simulation. Higher Education Press, Beijing. https://doi.org/10.1007/978-3-642-18101-6

[39] Čelikovsky, S. and Chen, G. (2005) On the Generalized Lorenz Canonical Form. Chaos Solitons \& Fractals, 26, 1271-1276. https://doi.org/10.1016/j.chaos.2005.02.040

[40] Shu, Y., Zhang, F. and Yang, H. (2011) Analysis of the Properties of a New Hyper-Chaotic System. Journal of Sichuan University, 48, 11-17. 